\title{
Umweltprobleme als Thema der Außenpolitik: Mexiko - USA
}

\author{
Von Günther Maihold
}

Während seines ersten Staatsbesuchs in den USA vom 2. bis 5. Oktober 1989 nannte der mexikanische Staatspräsident Carlos Salinas de Gortari bei seiner Rede vor dem amerikanischen Kongreß vier Themen, die die Agenda der bilateralen Beziehungen bestimmen: Handel, Migration, Drogen und Umwelt. Gleichzeitig sprach er von einer neuen Ära der Beziehungen, einer Ära der Kooperation und Freundschaft.1

Die herzliche und freundschaftliche Aufnahme des mexikanischen Präsidenten in den USA bezeichnet eine gewandelte Beziehung, die - historisch belastet und von aktuellen Problemen überlagert - einen in großem Maße statusorientierten Charakter besitzt. Unter dem Druck der notwendigen Strukturanpassungen im Gefolge der Schuldenkrise sowie der Öfnung des mexikanischen Marktes für ausländische Anleger und Produkte durch den GATT-Beitritt im Jahre 1986 hat sich die Kooperation zwischen Mexiko und USA auf einer Arbeitsebene versachlicht, die sich von den früheren Auseinandersetzungen um nationale Symbolik deutlich unterscheidet. Unter dem Stichwort der Modernisierung hat Mexiko den Weg einer handelspolitischen Reorientierung auf die USA eingeschlagen, der die früheren Versuche zu einer Diversifizierung der Außenbeziehungen revidierte. 2

Nicht ohne Grund ist daher von einer "stummen Integration"3 gesprochen worden, die mit dem Charakter der ökonomischen Beziehungen und der Migration begründet wird. Obwohl das Angebot der USA an Mexiko, dem nordamerikanischen gemeinsamen Markt beizutreten, von der mexikanischen Regierung abgelehnt wurde, stellt sich die Frage, inwieweit nicht schon de facto diese Integration vollzogen ist. Es geht somit gar nicht mehr darum, wie wünschenswert die Einbeziehung in den gemeinsamen nordamerikanischen Markt ist, sondern ob diese noch reversibel und von Mexiko hinreichend steuerbar ist, um mögliche Vorteile zu realisieren. 4 So absorbieren die USA bereits $60 \%$ des mexikanischen Außenhandels, während Mexiko der drittgrößte Handelspartner der USA ist.

1 Vgl. den Text der Ansprache von Carlos Salinas de Gortari vor dem amerikanischen Kongreß, in: La Jomada, 5. Oktober 1989, S. 14-16.

2 Vgl. insbesondere die Außenpolitik unter dem Präsidenten Luis Echeverría (1970-1976) und López Portillo (1976-1982).

3 Vgl. Jorge Castañeda/Robert Pastor, México: Elfu turo en juego, México, 1987, S. 146ff.

4 Vgl. Jorge Castañeda/Robert Pastor, Límites en la Amistad. México y Estados Unidos, México 1989, S. 300. 
Die illegale Wanderung der mexikanischen Arbeiter in die USA hat sich zu einem Problem der amerikansichen Innenpolitik entwickelt, was schon daraus ersichtlich wird, daß die sogenannten hispanics heute die größte ethnische Minderheit in den USA darstellen. Die Immigrationsgesetzgebung in Gestalt der Ley Simpson-Rodino vom 6. November 1986 war als Versuch gedacht, die Kontrolle über die Grenzen wiederherzustellen.5 Allerdings blieb der erwartete Abschreckungseffekt nur eine kurzfristige Erscheinung, die schon bald von der Routine wieder abgelöst wurde. 6

An der gemeinsamen Grenze zwischen Mexiko und den USA mit einer Länge von 3.597 $\mathrm{km}$ stehen sich sechs mexikanische Bundesstaaten und vier nordamerikanische Staaten gegenüber. Von besonderem Interesse ist dabei, daß die Grenze USA - Mexiko der einzige Ort in der Welt ist, an dem Nord und Süd bzw. Industrie- und Entwicklungsland direkt aufeinander treffen. Die stark von nationalistischen Untertönen getragene Debatte um die Kontrolle dieser Grenze bezog sich historisch betrachtet vor allem auf die Sicherung der nationalen Souveränität, so $\mathrm{da} ß$ es auf den ersten Blick überraschen muß, da $\beta$ in diesen Auseinandersetzungen Umweltprobleme Bedeutung gewinnen konnten. Den grenzüberschreitenden Umweltproblemen gelang es zwar in Einzelfällen, einen Spitzenplatz im Rahmen der bilateralen Verhandlungen auf hoher Ebene einzunehmen, im allgemeinen wurden sie jedoch als ein beispielhaftes Modell binationaler Kooperation behandelt, da sie eher "weiche" als sogenannte "harte" - d.h. krisenverursachende - politische Issues beinhalten. 7

Betrachtet man genauer die verschiedenen Umweltprobleme, die in der Außenpolitik der beiden Staaten diskutiert wurden, so stellt sich die Frage, wie es umweltpolitischen Themen gelingen kann, sich als sog. soft issues gegen die statusorientierten klassischen Bereiche der Außenpolitik durchzusetzen. In theoretische Termini übersetzt, ist damit das grundsätzliche Problem angesprochen, ob der Weg zu einer Lösung der globalen Umweltprobleme eher über die Definition einer Umweltaußenpolitik oder die Neugestaltung der internationalen Umweltbeziehungen führt. Der Fall der Beziehungen zwischen Mexiko und den USA kann in dieser Hinsicht insofem als gutes Beispiel dienen, da sich gerade die Diskussion um die internationale Lösung der Umweltprobleme in enger Verknüpfung von Umwelt- und Entwicklungspolitk artikuliert hat.

5 Die Aussage "We have lost control of our borders" kann als gemeinsame Parole der Gesetzesvorschläge Simpson-Mazzoli und Simpson-Rodino angesehen werden, gleichzeitig fungierte sie als Slogan im Wahlkampf Reagan vs. Carter.

6 Vgl. Jorge Bustamante, Migración de indocumentados de México a Estados Unidos, México 1988, S. 13f.

7 Vgl. Donald L. Wyman, Dependance and Conflict in US - Mexican Relations, 1920 - 1975, in: Robert L. Paarlberg/Eul Park/Donald L. Wyman (eds.), Diplomatic Dispute: US-Conflict with Iran, Japan and Mexico, Cambridge 1978, pp. 83-142. 


\section{Staatenwelt und Weltökologie}

Die Diskussion intemationaler Umweltprobleme hat sich unter dem Eindruck der vielfältigen Bedrohungen, die aus der globalen Sichtweise für die Zukunft der Menschheit ableitbar sind, von der zunächst dominanten Perspektive des Umweltschutzes zu einer grundsätzlicheren Sichtweise in Gestalt aktiver Umweltpolitik und Umweltgestaltung erweitert. Gerade in dieser Hinsicht hat der Interdependenzgedanke und die Verantwortung internationaler Organisationen große Bedeutung erlangt, da von diesen Instanzen die erfolgreiche Lösung der globalen Umweltprobleme erwartet wird, während die begrenzte Einsicht auf nationalstaatlicher Ebene als Hindemis für den Fortschritt einer internationalen Umweltpolitik angesehen wird. Daher ist der Versuch unternommen worden, jenseits der Konflikformationen Nord/Süd und Ost/West "unsere gemeinsame Zukunft"8 auf der Basis der Förderung eines gemeinsamen Verstehens und Verantwortungsbewußtseins zu sichern, um durch die Wahrnehmung verbindender Interessen das Überleben der Menschheit zu sichem.

Dieser idealistische Weg, den ökologischen Frieden ${ }^{9}$ zu sichern, gerät jedoch in Gefahr, an denselben Grenzen zu scheitem, die für den idealistischen Ansatz in der internationalen Politik generell gelten. 10 Eine vergleichbare Situation ergibt sich für die Entwicklung eines Umweltvölkerrechtes, das sich bisher weitgehend durch seine soft law-Komponente auszeichnet.

Als Alternative hierzu soll im folgenden ein den Interdependenzgedanken berücksichtigender Ansatz einer Umweltaußenpolitik vorgestellt werden, der die Begrenzungen des idealistischen Ansatzes und des sich auf das Völkerrecht beschränkenden Denkens überwinden kann.

\section{I.1. Umweltaußenpolitik oder internationale Umweltbeziehungen?}

Ausgehend von den Deklarationen der Umweltkonferenz der Vereinten Nationen in Stockholm 1972 hat sich eine Tendenz in der Umweltdiskussion entwickelt, die die transnationale und globale Verflechtung betont und gerade von der Neugestaltung der internationalen Umweltbeziehungen den Fortschritt für eine Neuordnung der Beziehungen von ökonomischer Entwicklung, technischem Wandel und Umweltsicherheit erwartet. Eine Durchsicht

8 So der Titel des Berichtes der Weltkommission für Umwelt und Entwicklung (BrundtlandBericht), deutscher Text herausgegeben von Volker Hauff, Greven 1987.

9 Vgl. Klaus Michael Meyer-Abich: Wege zum Frieden mit der Natur. Praktische Naturphilosophie für die Umweltpolitik. München 1984.

10 Vgl. Reinhard Meyers: Weltpolitik in Grundbegriffen. Bd. I, Ein lehr- und ideengeschichtlicher Grundriß. Düsseldorf 1979, S. 54ff. 
der Empfehlungen der Stockholmer Konferenz ${ }^{11}$ läßt deutlich erkennen, daß intemationalen Gremien der Vorzug bei der Bewältigung der globalen ökologischen Herausforderungen gegeben wird. Hinzu kommt, daß es den Entwicklungsländem schon im Vorfeld der Stockholmer Konferenz gelang, einen erweiterten Umweltbegriff durchzusetzen, der auch die soziale Umwelt einbezieht.12 Die Uberlappung von Entwicklungsinteressen und Umweltpolitik hat sich seitdem auf internationalen Konferenzen verstärkt ${ }^{13}$, zunehmend wird die Umweltdiskussion auf dieser Ebene von allgemeinpolitischen Forderungen überdeckt. Nicht ohne Grund ist daher vom "grenzenlosen Versagen" der intemationalen Umweltpolitik, wie sie sich vor allem im Rahmen des Umweltprogrammes der Vereinten Nationen (UNEP) konkretisierte, gesprochen worden. 14

Demgegenüber ist jüngst die Forderung nach einer nationalstaatlichen Umweltaußenpolitik aufgestellt worden, die gerade die Überschätzung der Durchsetzbarkeit und Reichweite supranationaler Lösungsansätze in Rechnung stellt, und stattdessen als Basis die Eigeninteressen des Staates formuliert. Als Umweltaußenpolitik wird "die Gesamtheit der Aktivitäten eines Staates bei der Wahmehmung seiner Interessen bezüglich umweltpolitischer Fragen gegenüber anderen Grundeinheiten der internationalen Politik (vor allem Nationalstaaten und internationalen Organisationen)"15 definiert. Es liegt daher im Sinne einer nationalen Umweltaußenpolitik, das eigene Territorium soweit als möglich vor grenzüberschreitenden Belastungen zu schützen und gegebenenfalls möglichst viele Schadstoffe ins Ausland abzuschieben. Dem Argument, solche Umweltbelastungen würden mittelbar wieder auf dis eigene Bevölkerung einwirken und seien deshalb keine sinnvolle politische Strategie16, steht zum einen der internationale Mülltourismus im Nord/Süd-Kontext und zum anderen das machtpolitische Kalkül des Nationalstaates entgegen, das weitgehend langfristiger politischer Sichtweise entbehrt.

Die Strategie einer Umweltaußenpolitik, nach gemeinsamen Umweltinteressen mit den Nachbarländem zu suchen ${ }^{17}$, gerät dort an ihre Grenze, wo die Kostenbelastung durch den Umweltschutz für die nationalen Volkswirtschaften stark voneinander abweicht. Diese Verzerrung der Kostenstruktur legt zwar eine internationale Harmonisierung von Normen

11 Vgl. Michael Kilian, Umweltschutz durch intemationale Organisationen. Die Antwort des Völkerrechtes auf die Krise der Umwelt? Berlin 1987, S. 250ff.

12 Vgl. den sog. Founex Raport, Environment and Development, in: Intemational Conciliation, Nr. 586/Januar 1972.

13 Vgl. dazu kritisch Kilian, op. cit., S. $271 \mathrm{ff}$.

14 Vgl. Elmar Römpczyk, Intemational Environmental Policy as a Challenge to the Politics of Development, Bonn 1987, pp. 9ff.

15 Vgl. Volker Prittwitz, Umwelt und Außenpolitik, in: Aus Politik und Zeitgeschichte B 42/83, 22. Oktober 1983, S. 17.

16 Vgl. Volker Prittwitz, Umweltaußenpolitik, Grenzïberschreitende Luftverschmutzung in Europa. Frankfurt 1984, S. 15.

17 Prittwitz op.cit., S. 19. 
und Standards nahe, löst aber nicht die Probleme der unterschiedlichen Leistungsfähigkeit, wenn beispielsweise Staaten wie die USA und Mexiko aufeinandertreffen, die dem Norden bzw. dem Süden angehören. Weiterhin stellen Umweltpolitik und Außenpolitik getrennte politische Sphären dar, die auch mit unterschiedlichen Instrumenten arbeiten: Während die Umweltpolitik sich vor allem durch die Maßnahmenkosten auszeichnet, ist die klassische Außenpolitik in viel stärkerem Maße statusorientiert. Zudem setzt die Integration umweltpolitischer Themen in die Außenpolitik ein politisch artikuliertes Interesse an diesen Fragestellungen in den interagierenden Staaten voraus, was jedoch nicht notwendigerweise gegeben ist. Gerade wenn man den hier vorliegenden Fall der Beziehungen zwischen Mexiko und den USA vor Augen hat, wird deutlich, $\mathrm{da} ß$ die Voraussetzung eines politisch wirksamen Umweltbewußtseins, zumindest was die mexikanische Seite anbelangt, nicht vorliegt. Die geforderte Parallelität von Umweltinnen- und Umweltaußenpolitik kann somit nicht zur grundsätzlichen Bedingung eines Konzeptes von Umweltaußenpolitik erhoben werden 18 , da damit ein idealistisches Element in die Analyse eingebaut wird, das eher voluntaristischen Charakter besitzt. Zudem ist das Verhältnis von Innen- und Außenpolitik hinreichend kompliziert, um eine einfache Entsprechung dieser Politikbereiche unterstellen zu können.19

Die Trennlinie verläuft vielmehr zwischen den sog. high and low-politics 20 , also zwischen den als technisch oder soft eingestuften politischen Issues einerseits und jenen umstrittenen und an politischen Statusfragen orientierten Themen andererseits. In dem uns interessierenden Fall erfolgte die Diskussion der Umweltproblematik auf der Ebene der Staatspräsidenten meist weniger unter dem Gesichtspunkt gemeinsamer Umweltinteressen, sondern war geprägt von nationalstaatlichem Souveränitätsdenken. Umweltschutzziele erhalten auch unter dem Gesichtspunkt einer Umweltaußenpolitik meist erst dann systematische Bedeutung, wenn sie in "Pakete" der Außen- und Wirtschaftspolitik eingebunden werden; damit ist eines jener Elemente angesprochen, die von der funktionalistischen Integrationstheorie hervorgehoben wurden: das Package-dealing eines "kultivierten spill-over".21

In diesem Punkt wird offenbar, daß das Konzept einer Umweltaußenpolitik weithin hybriden Charakter besitzt: Obwohl es sich vordergründig der realistischen Schule des Machtstaates verbunden gibt, ist es gleichwohl weiterhin dem idealistischen Gedankengut

18 Prittwitz spricht an diesem Punkt zwar nur von "Glaubwürdigkeit" (S. 205), aber gleichzeitig gerät mit dieser Beurteilung sein gesamtes Konzept ins Wanken.

19 Vgl. Günther Maihold, Das Verhältnis von Innen- und Außenpolitik in Entwicklungsländem: Der Fall Mexiko, in: Verfassung und Recht in Übersee Bd. 20/1987, S. $220 f$.

$20 \mathrm{Vgl}$. die aus der funktionalistischen Integrationstheorie erfolgende Begründung dieser Unterscheidung bei David Mitrany, A working peace system, London 1947, pp. $28 \mathrm{ff}$.

21 Vgl. Joseph S. Nye, Ein Vergleich gemeinsamer Märkte, in: G. Dokeker/F. Veitl, Regionalismus und regionale Integration. Zur Theorie der regionalen Integration. Zur Theorie der regionalen Integration, Frankfurt 1981, S. 193. 
verwandt, indem es Notwendigkeiten einer internationalen Umweltpolitik auf der Grundlage objektiver und globaler Verpflichtungen anerkennt. Es findet somit nur eine strategische Verschiebung des politischen Ansatzes statt, indem die geringen Erfolge der internationalen Umweltbeziehungen zum $A$ nlaß genommen werden, um nunmehr den Nationalstaat als Hauptakteur für den Erfolg der vordefinierten Ziele zu definieren.

Ein Element, das der Perspektive der internationalen Umweltbeziehungen entgeht und das stattdessen vom Ansatz der Umweltaußenpolitik hervorgehoben wird, ist die Bedeutung der subnationalen Einheiten 22 bei der Regelung grenzüberschreitender Umweltprobleme. Zwar werden diese Instanzen meist nicht in die offiziellen zwischenstaatlichen Verhandlungen eingebunden, sie stellen aber gleichwohl eine wichtige Dimension auf der Ebene der lowpolitics dar, um spezifische Fragestellungen lösen zu können.

Diese "Mikro-Diplomatie"23 artikuliert sich vor allem in funktionalen Bereichen der Außenpolitik und ist Ausdruck der lateralen Beziehungen subnationaler Aktoren zwischen Gesellschaft und Gesellschaft der interagierenden Staaten, die neben den horizontalen linkages zwischen den Regierungen und den integrativen oder supranationalen Prozessen, an denen die Staaten beteiligt sind, das Netz der internationalen Beziehungen bilden. 24 In diesem Sinne wird ein Interdependenz-Modell verfolgt, das sich in der internationalen Politik auf Situationen bezieht, die durch reziproke Effekte zwischen Ländern oder zwischen Aktoren unterschiedlicher Länder definiert sind.25 Eng verbunden mit diesem Konzept der Interdependenz ist die Einsicht in asymmetrische Beziehungen, die zur Verletzbarkeit im Sinne des Nachteils eines Aktors führt, der weiterhin Kosten für externe Ereignisse hinnehmen muß, obwohl er seine Politik entsprechend verändert hat. 26 Gerade das Beispiel Mexiko - USA verdeutlicht diese Situation, die sich auch im Umweltbereich konkretisiert hat.

Eine sinnvolle Konzeption von Umweltaußenpolitik kann sich daher nicht auf die inhaltliche Füllung des Nationalstaatspostulates der realistischen Schule beschränken, sondern bedarf der Ergänzung durch den Interdependenzgedanken, der den relativen Handlungsspielraum des Staates in der internationalen Politik erkennbar macht sowie die Abgrenzung von high und low politics gestattet.

22 So auch Prittwitz, op. cit., S. 23.

23 Vgl. Ivo D. Duchacek, The Territorial Dimension of Politics. Within, Among, and Across Nations, Boulder 1986, S. 226ff.

24 Vgl. Wolfram F. Hanrieder, Dissolving Intemational Politics: Reflections on the Nation - State, in: The American Political Science Review Vol. 72/1978, p. 1278.

$25 \mathrm{Vgl}$.Robert O. Keohane/Joseph S. Nye, Power and Interdependence: World Politics in Transition, Boston 1977, p.8.

26 Vgl. Keohane/Nye, op. cit., p. 28. 


\section{I.2. Ökologische Sicherheit und nationale Souveränität}

Das Prinzip Nr. 21 der Deklaration der Umweltkonferenz von Stockholm spiegelt die Problematik des Verhältnisses von internationaler Kooperation und nationaler Souveränität im Umweltbereich klar wieder: "Gemäß der Charta der Vereinten Nationen und den Prinzipien des Internationalen Rechtes haben die Staaten das souveräne Recht zur Ausbeutung ihrer eigenen Hilfsquellen nach Maßgabe ihrer eigenen Umweltpolitik und die Verpflichtung, dafür zu sorgen, daß durch Tätigkeiten innerhalb ihres Hoheits- und Kontrollbereiches der Umwelt in anderen Staaten oder Gebieten außerhalb ihres nationalen Hoheitsbereiches kein Schaden zugefügt wird."27 Mit diesem Dokument wurde der erste bedeutende Fall des soft law im Umweltschutz begründet, der in einem geradezu artistischen Balanceakt versucht, das souveräne Recht der Ausbeutung der natürlichen Ressourcen mit der Verantwortlichkeit der Staaten zu verbinden.

Wie in Stockholm, so sind bis heute noch nicht Grundsätze für Haftung und Schadenersatz auf internationaler Ebene konsensfähig, so daß nach wie vor gewisse Beiträge des Völkergewohnheitsrechtes die Basis für die Weiterentwicklung eines Umweltvölkerrechtes bilden. 28 Aus dem Prinzip der guten Nachbarschaft (Art. 74 der UN Charta) hat sich bis heute noch kein konkreter Individualschutz ableiten lassen, obwohl in den sog. UNEP Guidelines von 1978 erstmals die umweltbezogene Benachteiligung von Grenznachbam als Thema für ein internationales Haftungsrecht vorgeschlagen wurde.29 Darüber hinaus sind Beiträge zur Ausbildung eines internationalen Umweltschutzrechtes auf dem Weg über bilaterale Verträge, wie die durch Notenwechsel getroffene Vereinbarung zwischen Mexiko und den Vereinigten Staaten vom 30. August 1973 über den Salzgehalt des Colorado, denkbar. Das bisher wenig entwickelte Vertragsvölkerrecht bedarf in seiner Anwendung auf die Umweltproblematik eines multilateralen Ansatzes, was gleichzeitig die Bedeutung internationaler Organisationen verstärkt. Allerdings sind entsprechende Bemühungen bisher über Programmsätze und Organisationszielbestimmungen nicht hinausgekommen, so daß die Masse dieser vor- und außerrechtlichen Aktivitäten der intemationalen Organisationen

27 Vgl. The Results of Stockholm, Les Résultats de Stockholm, Stockholmer Resultate, Beiträge zur Umweltgestaltung, Heft A 10, Berlin 1973.

28 Vgl. den Trial-Smelter-Fall (1938/41) und den Gut-Dam-Fall (1967/68), die sich beide im Grenzbereich Kanada-USA ereigneten, der erstere hinsichtlich der Luftverschmutzung, der zweite in bezug auf Flutschäden. Die intemationale Schiedsgerichtsbarkeit entschied damals, da $B$ jeder Staat die Verantwortung dafür trägt, auf seinem Territorium keine Maßnahmen zuzulassen, die Schäden auf dem Gebiet benachbarter Staaten verursachen bzw. daß er gegebenenfalls nach den Grundsätzen der Staatenhaftung dafür verantwortlich gemacht werden kann.

29 Vgl. Thomas Oppermann, "Gute Nachbarschaft" im internationalen und europäischen Umweltrecht, in: Wilhelm Grewe (Hg.): Europäische Gerichtsbarkeit und nationale Verfassungsgerichtsbarkeit, Festschrift für Hans Rutscher, Baden-Baden 1983, S. 301-318. 
eher den Charakter meinungsbildender Maßnahmen besitzen, die durch Rechtsvergleichung und -angleichung weiterzuentwickeln sind.

Zusammen mit der für das Umweltvölkerrecht kennzeichnenden sozialen Komponente (sozialer Umweltbegriff/Verbindung Entwicklung-Umwelt) stellen sich einer Weiterentwicklung folgende Probleme: Zum einen die unterschiedlichen Entwicklungsstufen der Staaten, ihre spezifischen geographischen Situationen und Rechtsverständnisse, sowie die abweichenden Rechtstraditionen.30

Von manchen Autoren wird das Verursacherprinzip als die einleuchtendste und einfachste Grundmaxime betrachtet, wobei diese jedoch meist auf eine einfache Kostenzurechnungsgrundlage verkürzt wird. Mit der Anwendung dieses umweltpolitischen Konzepts auf internationaler Ebene sind aber eine Fülle von Problemen verbunden: Unabhängig von der Frage, ob auf der Grundlage dieses Prinzips es nicht dem Staat ausgesprochen leicht gemacht wird, die Umweltkosten auf den Verursacher abzuwälzen, gerät dieses Konzept in große Schwierigkeiten bei der Beteiligung von Entwicklungsländern, deren Leistungspotential begrenzt ist. Es ist zu fragen, inwieweit nicht eine Art von 'internationalem Gemeinlastprinzip' in Gestalt eines Fonds besser geeignet ist, zu einer Beseitigung von Umweltschäden beizutragen.31 So sollte das Verursacherprinzip generell dahingehend interpretiert werden, daß der Verursacher für die Vermeidung und/oder Beseitigung von Umweltbelastungen nach Maßgabe internationaler Qualitätsziele verantwortlich ist und daß ihm gegebenenfalls seitens der internationalen Gemeinschaft zumindest Beihilfe für die Kosten angeboten werden sollte.

Notwendigerweise beinhaltet dies die Festlegung übergreifender Umweltstandards und die Akzeptierung einer präventiven Umweltgestaltung, um die notwendigen Grundlagen für ein solches Modell zu schaffen. Obwohl die Praxis der internationalen Umweltpolitik von der Harmonisierung von Umweltnormen dominiert war, ist auf internationaler Ebene kein Fortschritt in der Frage des Vollzugsdefizits erzielt worden. Zudem hat sich gerade der Versuch, Umweltschutzstandards international anzugleichen, als Hindernis für die Weiterentwicklung des Umweltvölkerrechtes erwiesen, da es nicht gelungen ist, die komplizierte technologische und technische Materie in völkerrechtliche Normen umzusetzen.32

30 Vgl. Michael Kilian, Umweltschutz durch intemationale Organisationen. Die Antwort des Völkerrechtes auf die Krise der Umwelt? Berlin 1987, S. 54.

31 Vgl. Thomas Oppermann, Gesetzte Normen des Völkerrechts zum Umweltschutz und die Grundlagen und Verfahren ihres Erlasses, in: V. Götz/D. Rauschning/G. Zieger (Hg.), Umweltschutz und internationale Wirtschaft, Rosenheim 1975, S. 18.

32 Vgl. Prittwitz op. cit. (APUZG) S. 18 sowie Helmut Steinberger, Beachtung und Durchsetzung völkerrechtlicher Umweltschutznormen, in: Götz/Rauschning/Zieger op. cit. $26 f$. 
Es ist daher nicht verwunderlich, daß sich die ungeklärte Beziehung von ökologischer Sicherheit und nationaler Souveränität in der Diskussion um zwei Begriffe niederschlägt, die für die internationale Umweltdiskussion maßgeblich geworden sind: "Shared natural ressources" und "Common heritage of mankind". Während der erste Begriff in das Umweltvölkerrecht bereits übernommen wurde, da er der Bestätigung der jeweiligen nationalen Souveränität über die Ressourcen dient, ist der Begriff des "common heritage" weitaus umstrittener. Dem Völkervertragsrecht entsprungen, ist er in Verdacht geraten, eine jener Generalklauseln zu sein, hinter der sich ungelöste politische Probleme verbergen33; gleichwohl taucht dieses Konzept im Seerecht, im Mondvertrag sowie im Umweltvölkerrecht auf. Der Grundsatz vom Menschheitserbe hat sich zu einem Schlüsselbegriff in der Auseinandersetzung um eine neue Weltwirtschaftsordnung entwickelt, so daß vor allem der nutzungsrechtliche Aspekt zwischen Nord und Süd umstritten ist, während das gebietsrechtliche, das sicherheitspolitische, das wissenschaftspolitische und das ökologische Element schon 1970 außer Diskussion waren.34 Es ist bezeichnend, daß auch das Umweltvölkerrecht gerade an diesem Punkt in eine politisch begründete Aporie gerät und damit zur Definition eines gangbaren Weges zwischen den Notwendigkeiten globaler ökologischer Sicherheit und den Realitäten nationalstaatlich begrenzter Umweltgestaltung wenig beizutragen hat. Die konkrete Formulierung nutzungsrechtlicher Bestimmungen über die Verwendung natürlicher Ressourcen bleibt daher ebenso wie die Harmonisierung von Umweltstandards auf internationaler Ebene blockiert, so da $\beta$ die Problematik politisch auf die bilaterale Ebene zurückverwiesen wird.

Es ist somit am gewählten Beispiel zu diskutieren, inwieweit die grundsätzliche Problematik der internationalen Verfügbarkeit umweltpolitischer Instrumente aus den bilateralen Beziehungen abgeleitet werden kann, zumal wenn sich diese unter den spezifischen Bedingungen der Kooperation zwischen einem Industrie- und einem Entwicklungsland ergeben.

\section{Die Struktur der außenpolitischen Beziehungen zwischen Mexiko und den USA}

In seinem Roman Gringo Viejo schreibt der mexikanische Schriftsteller Carlos Fuentes, die Nordgrenze Mexikos sei weniger als Wunde denn als Narbe zu verstehen. 35 Diese Beschreibung verweist auf die historischen Belastungen in den Beziehungen Mexiko-USA, ist aber gleichzeitig auch als Hinweis darauf anzusehen, daß die Konfliktträchtigkeit dieser

33 Vgl. Adrian Bueckling: Zur juristischen Substanzlosigkeit des Begriffs: Gemeinsames Erbe der Menschheit", in: Deutsche Richterzeitung August 1981, S. 288-294.

34 Vgl. Wilhelm Kewenig, Common heritage of mankind - Politischer Slogan oder völkerrechtlicher Schlüsselbegriff?, in: Staatsrecht-Völkerrecht-Europarecht, Festschrift für Hans-Jürgen Schlochauer, Berlin 1981, S. 385-406.

35 Vgl. Rolando Cardera/Carlos Fuentes/Lorenzo Meyer/Jorge Castañeda, Frontera Norte: la cicatriz y la herida, in: Nexos No. 140/August 1989, S. 29. 
Grenze zwischen Nord und Süd inzwischen größerer Routine gewichen ist. Aus der Sicht der USA läßt sich zumindest hinsichtlich der Umweltproblematik eine vergleichbare Belastung an der Grenze zu Kanada feststellen, wobei aufgrund der ökonomischen Unterschiede Mexiko und Kanada nicht gleichzustellen sind. Allerdings hat sich in beiden Fällen eine Umweltdiplomatie entwickelt, die deutlich macht, daß mit der Zunahme in der Dramatik der Umweltsituation eine an Prinzipien orientierte Lösung immer schwieriger wird. Ebenso wie für Mexiko gilt auch für den kanadischen Fall, daß es den USA nicht gelungen ist, eine einheitliche Politik zu entwickeln, die den langfristigen Interessen beider Länder gerecht würde.36

Die Struktur der Beziehungen zwischen Mexiko und USA läßt sich anhand dreier Grundelemente beschreiben, die gleichzeitig die Verhandlungsmuster auf verschiedenen Ebenen bestimmen:

1. Die territoriale Grenze, welche weiterreichende Implikationen militärstrategischen Charakters besitzt und gleichzeitig für Mexiko eine Begrenzung seiner de facto Autonomie bedeutet;

2. eine Machtasymmetrie, die Mexiko in die schwächere Position im Rahmen der Verhandlungen bringt;

3. die ökonomische und technologische Abhängigkeit von den USA, die die Verletzbarkeit Mexikos hinsichtlich von Entscheidungen der US-Regierung oder transnationaler Unternehmen berührt. 37

Diese strukturelle Asymmetrie der Interdependenz bildet den Hintergrund für die stärker an politisch-konjunkturellen Gesichtspunkten orientierte Politik beider Länder. So läßt sich bis 1971 eine am Konzept der "Politik der guten Nachbarschaft" ausgerichtete Routinebeziehung nachweisen, welche jedoch seitens Mexikos zunehmend fragmentiert wahrgenommen wird. Während vorher das nordamerikanische Außenministerium die Beziehungen mit Mexiko monopolisierte, wird zunehmend auch durch den Kongreß Mexiko zu einem kontroversen innenpolitischen Thema 38 erhoben. Mit der Erdölkrise und der Entdeckung der mexikanischen Erdölvorkommen wuchs Mexiko ab 1977 hohe Priorität in den amerikanischen Außenbeziehungen zu, während wiederum Mexiko die politische Lage zu einer Neugestaltung der "special relationship" zu seinen Gunsten nutzen wollte.39 Diese Strategie scheiterte jedoch an der ökonomischen Krise des Landes sowie den Entwicklungen in Zen-

$36 \mathrm{Vgl}$. John E. Carroll, Environmental Diplomacy. An examination and a prospective of CanadianU.S. Transboundary Environmental Relations, Ann Arbor 1986, p. 304.

37 Vgl. Mario Ojeda, El futuro de las relaciones entre México y los Estados Unidos, in: C. Tello/C. Reynolds (Selecc.), Las relaciones México - Estados Unidos, México 1981, S. 384ff.

38 Vgl. Donald L. Wyman, The United States Congress and the Making of US Policy towards Mexico, University of San Diego: Working Papers in US-Mexican Studies 13/1981, pp. 4ff.

39 Vgl. Robert E. Scott, National Development and Mexicos Foreign Policy, in: Intemational Journal Vol. 37/1981-82, S. 57. 
tralamerika, wodurch der Druck der USA auf Mexiko wiederum zunahm, da insbesondere die Rolle Mexikos in der Contadora-Gruppe nicht gebilligt wurde. Das Ansteigen der Migration 1982 und die verstärkte Aufmerksamkeit für das Drogenproblem ab 1986 führte zur Ausarbeitung einer informellen parallelen Agenda in der nordamerikanischen Außenpolitik, die sich insbesondere auf Themen bezieht, die seitens Mexikos zum ausschließlichen Souveränitätsbereich gerechnet werden.40 Die Hearings, die der amerikanische Kongreß 1986 zum Thema Mexiko durchführte, wurden in Mexiko als Versuch interpretiert, neue Spielregeln in die binationalen Beziehungen einzubringen.

Für die Frage der grenzüberschreitenden Umweltprobleme sowie die konkrete Ausgestaltung der Zusammenarbeit in der Grenzregion bedeutete dies eine qualitative Veränderung: Viele der zwischen beiden Ländern strittigen politischen und ökonomischen Themen wurden seitens der USA unter dem Gesichtspunkt der Bewahrung der natürlichen Ressourcen vorgebracht, so daß erst der Besuch des mexikanischen Präsidenten Miguel de la Madrid im August 1983 eine Bereinigung der bilateralen Beziehungen brachte, die eine sachbezogene Bearbeitung der Umweltprobleme ermöglichte. Gleichwohl brachten die USA, insbesondere in Hinsicht auf die Drogenproblematik den Gedanken einer "Militarisierung" der Grenze ins Spiel, der von der mexikanischen Seite mit großer Sensiblität aufgenommen wurde. 41

Gerade die Diskussion bezüglich einer Neuordnung der Grenzregion gehört zu den für Mexiko besonders aufmerksam verfolgten Themen. Wenn man sich vor Augen führt, daß das Grenzgebiet, die sog. borderlands, nicht nur einen geographischen Raum oder eine rechtlich politische Einheit bezüglich einer Grenzlinie darstellen, sondem auch als eigenständige politisch kulturelle Einheit zu verstehen sind42, in der sich eigene Traditionen herausbilden, dann sind die politischen Folgen eines Vorhabens wie die Militarisierung der Grenze nicht abzusehen. Durch die Wanderungsbewegungen an der Grenze, die sich seit dem Ende der 70er Jahre auch als eigenständiger Wirtschaftsraum entwickelt hat, sind die Probleme auf beiden Seiten der politischen Trennlinie gleichermaßen gewachsen; dies gilt in besonderem Maße auch für die Umweltprobleme, welche aus den erforderlichen Infrastruktureinrichtungen erwachsen.

40 Hiermit sind insbesondere Angriffe auf Angehörige der mexikanischen Regierung hinsichtlich ihrer Verwicklung in Komption und Drogengeschäfte gemeint; vgl. Mario Ojeda, La doble agenda en las relaciones entre México y los Estados Unidos, in: Gerardo Bueno (Comp.) MéxicoEstados Unidos 1986, México 1987, S. $21 \mathrm{ff}$.

41 Vgl. Guy F. Erb/Catherine Thorup, México y Estados Unidos: estrategias nacionales e intereses bilaterales, in: Gerardo Bueno (Comp.), op. cit., S. 81.

42 Vgl. Ellwyn Stoddard, US-Mexico Borderlands Studies, El Paso 1974, S. 17. 


\section{II.1. Grenzüberschreitende Umweltprobleme: Mexiko - USA}

Es ist bezeichnend, daß sich der erste Konflikt zwischen Mexiko und den USA im Umweltbereich weniger aus der Beeinträchtigung natürlicher Ressourcen entwickelte, sondem der Frage der Nutzung intemationaler Gewässer entsprang. Sowohl der Colorado River wie auch der Río Bravo, die zusammen mit dem Río Tijuana Grenzflüsse zwischen Mexiko und den USA bilden, veränderten immer wieder ihren Lauf, so daß sich Gebietsansprüche beider Länder ergaben. Zwar wurde im Jahre 1852 der Grenzverlauf in der Mitte des Flußbettes vereinbart, aber die Auseinandersetzungen setzten sich insbesondere hinsichtlich eines Landstückes, El Chamizal, fort, das zwischen Ciudad Juárez, Chihuahua und El Paso, Texas gelegen ist. Obwohl Mexiko im Jahre 1964 schließlich dieses Gebiet zurückerhielt, war diese Streitigkeit der Auftakt für die Ausbildung einer binationalen Verhandlungsinstitution, die International Boundary Commission, die im Jahre 1889 ihre Arbeit aufnahm. Im Jahre 1928 wurde dieser Konsultationsmechanismus in die International Boundary and Water Commission umgewandelt, die die generelle Verantwortung für die Regelung der Wasserfragen erhielt. 43

\section{II.1.1. Nutzungsrechte und Wasserverschmutzung: Die Versalzung des Río Bravo}

Ihren Ursprung nahm diese Kontroverse in der Klage Mexikos im Jahre 1895, im Unterlauf weniger Wasser als in den Vorjahren zu erhalten. Die USA antworteten mit der sog. Harmon-Doktrin, daß sie nach intemationalem Recht nicht verpflichtet seien, Mexiko Wasser zur Verfügung zu stellen. Im Jahre 1906 wurde ein Vertrag über die Verteilung des Wassers des Río Bravo geschlossen, in dem Mexiko 74 Millionen Kubikmeter Wasser pro Jahr zugesichert wurden.44 Mit der Einrichtung der International Boundary and Water Commission im Jahre 1928 wurden vergleichbare Zuteilungsrechte auch für den Río Colorado sowie den Río Tijuana getroffen. Allerdings enthalten diese Regelungen konkrete Ziffern, die keinerlei Raum für zukünftige Bedürfnisse lassen, darüber hinaus wurden keine genauen Angaben über die Wasserqualität gemacht, so daß dieser Vertrag zur Quelle zukünftiger Konflikte zwischen den beiden Ländem wurde.

Der im Jahre 1961 plötzlich ansteigende Salzgehalt des Colorado River, der von Bauem aus Arizona verursacht wurde, bewirkte einen vollständigen Verlust der Emte im Tal von Mexicali (Mexiko) und rief den Protest der mexikanischen Regierung bei den USA wegen Nichteinhaltung des Vertrages von 1944 hervor. Die Lösung dieses Konfliktes, die von der

$43 \mathrm{Vgl}$. Stephen Mumme, State Influence in Foreign Policy Making: Water Related Environmental Disputes Along the United States - Mexico Border, in: The Westem Political Quarterly Vol. 38/1985, pp. 624ff.

44 Vgl. zu dieser Problematik César Sepúlveda: La frontera norte de México. México 1976, S. $93 \mathrm{ff}$. 
International Boundary and Water Commision vorgeschlagen wurde, sah den Bau eines Abwasserkanals vor, der die versalzten Gewässer direkt in den Golf von Kalifomien leiten sollte. Allerdings wurde dieses Wasser auf die Nutzungsquote Mexikos angerechnet, ein Mangel, der erst im Jahre 1972 durch eine Vereinbarung der Präsidenten Nixon und Echeverría beseitigt wurde. 45

Über diese Vereinbarung hinaus bleiben jedoch noch weitere binationale Umweltprobleme bestehen: Die Verteilung der Grundwasservorhaben, der Anstieg des Wasserbedarfs auf der mexikanischen Seite des Río Colorado sowie die nach wie vor nicht grundlegend beseitigte Versalzung des Colorado River. Der eingeschlagene Weg, über die Schaffung eines intergouvernementalen Organismus mit exekutiven und legislativen Funktionen grenzüberschreitende Umweltprobleme zu lösen, folgte in diesem Bereich einer Fall zu Fall-Strategie, die Mexiko in die schwächere Position brachte. Gleichwohl waren die USA, nicht zuletzt unter dem Eindruck der Stockholmer Umweltkonferenz von 1972 gezwungen, ihre diskriminierende und völkerrechtlich bedenkliche Haltung aufzugeben, die Wasserquote de facto zu senken. Der Erfolg der International Boundary and Water Commission als intergouvernementale Instanz läßt sich vor allem daran erkennen, daß sie inzwischen auch die Realisierung gemeinschaftlicher Unternehmen wie den Bau von Staudämmen und Kläranlagen übernommen hat.

\section{II.1.2. Verursacherprinzip und Abwasserregulierung: Tijuana und San Diego}

Die Klage der USA, Abwässer der Stadt Tijuana (Mexiko) verschmutzten die Strände von San Diego, führte in den Jahren 1984 und 1985 zu heftigen Kontroversen zwischen beiden Ländem. Obwohl beide Städte einen gemeinsamen Abwasserkanal in die Bucht von San Diego benutzen, verschärfte sich die Auseinandersetzung im Gefolge der zunehmenden Mengen aufgrund des Anwachsens der Städte. In einem Übereinkommen aus dem Jahre 1965 war die Beschickung einer Kläranlage in San Diego mit Abwässem von Tijuana vorgesehen, wenn die mexikanische Anlage nicht ausreichen sollte. Im Jahre 1986 kündigte San Diego diese Vereinbarung, da Tijuana über die paktierten Mengen hinaus Abwässer anlieferte. Das Ableiten der verschmutzten Gewässer durch Mexiko in den Río Tijuana führte zu Protesten seitens der amerikanischen Regierung, während die Stadtverwaltung von Tijuana für die Verschmutzung der Strände in San Diego Firmen verantwortlich machte.

Im Rahmen der binationalen Verhandlungen vertrat die amerikanische Seite unter der Regierung Reagan streng das Verursacherprinzip und weigerte sich, eine Lösung anzuneh-

45 Vgl. Herbert Brownell/Samuel D. Eaton: The Colorado River Salinity Problem with Mexico, in: The American Joumal of International Law, vol. 69/1975, pp. $255 \mathrm{ff}$. 
men, in der US-amerikanische Einrichtungen mit Finanzierung durch die Zentralregierung Abwässer von Tijuana behandelten. 46

Auf der Basis des 1983 zwischen Mexiko und den USA beschlossenen Agreement on Cooperation for the Protection and Improvement of the Environment in the Border Area sowie Anhang 1 zu dieser Vereinbarung bestanden die USA auf der strikten Einhaltung des Verursacherprinzips und sahen sich nicht in der Lage, Beihilfen für den Bau der notwendigen Abwasserregulierung und -behandlung zu leisten. Gerade an diesem Punkt wird deutlich, daß die in Art. 18 dieser Vereinbarung vorgesehene Regelung, vereinbarte Aktivitäten seien Gegenstand der verfügbaren finanziellen Möglichkeiten jeder Seite und der Anwendung der entsprechenden Gesetze und Richtlinien, zu einer Verzögerung der Umweltmaßnahmen führte. Darüber hinaus wurden die Grenzen gemeinsamer Umweltpolitik im Spannungsfeld unterschiedlicher finanzieller Potentiale und unterschiedlicher Entwicklung des Umweltbewußtseins erkennbar, da Mexiko ohne eigenständige Informationsgrundlage in diesem Kontext den Forderungen der USA gegenüberstand, ohne durchführbare Alternativen präsentieren zu können. Mexiko konnte sich nur verpflichten, aufgrund begrenzter Finanzen bis zum Jahre 2000 eine Kläranlage zu erstellen, die so gelegen ist, daß eine Verschmutzung der Strände von San Diego wegen der Meeresströmungen ausgeschlossen werde. Die einseitige Lösung, die Mexiko vorschlug, wird gemeinsamen Umweltinteressen nicht gerecht, obwohl sich die amerikanische Regierung zugute hielt, einen Präzedenzfall durch die strikte Beachtung des Verursacherprinzips vermieden zu haben. 47

\section{II.1.3. Luftverschmutzung und regionale Entwicklung: Der Konflikt um die Kupferproduk- tion}

Das im August 1983 von den Präsidenten Reagan und de la Madrid unterzeichnete Agreement between the United States and the United Mexican States on Cooperation for the Protection and Improvement of the Environment in the Border Area sieht für die Lösung der grenzüberschreitenden Umweltprobleme eine $100 \mathrm{~km}$ breite Zone innerhalb der beiden Länder vor, die auf der Basis von Gleichheit, Reziprozität und gegenseitigem Nutzen zur Verbesserung und Bewahrung der Umwelt durch einen von der jeweiligen Regierung genannten nationalen Koordinator verwaltet wird. Einen ersten Anwendungsfall für diese Agreements stellten die Luftbelastungen der Kupferproduktion in Schmelzöfen dar, die im

46 Vgl. Stephen MummelJoseph Nalven, Managing the Border Environment: Advances, Issues and Options, in: Oscar Martínez/Albert Utton/Mario Miranda Pacheco (eds.). One Border, Two Nations: Policy Implications and Problem Resolutions, Mexico 1988, pp. 246ff.

47 Vgl. Roberto Sánchez: La negociación de conflictos ambientales entre México y Estados Unidos, in: Frontera Norte Vol. 1/1989, S. 90ff. 
sog. grauen Dreieck (vgl. Fig. Nr. 2) gelegen ist und sowohl die nordamerikanischen wie die mexikanischen Produktionsstätten einschließt.

Die Konkurrenz zwischen den in Sonora und in Arizona angesiedelten Firmen entzündete sich zunächst an der Gewährung eines 450 Millionen Dollar Kredites der Weltbank im Juli 1982, der zur Modernisierung und Erweiterung der in Cananea angesiedelten Produktionsstätte dienen sollte. Angesichts der schwierigen Situation auf dem Kupfermarkt erhob sich seitens der nordamerikanischen Kupferproduzenten erhebliche Kritik, da eine Begünstigung der mexikanischen Produktion zu Lasten der USA befürchtet wurde. Insbesondere führten die nordamerikanischen Firmen Wettbewerbsnachteile an, da die mexikanischen Unternehmen nicht mit den Vorschriften und Umweltstandards belastet seien, denen sie unterworfen waren (Clean Air Act).48 Innerhalb kurzer Zeit bildete sich in den USA eine gemeinsame Front von Kongreßabgeordneten, Gewerkschaften und Umweltgruppen (Greenpeace, Sierra Club, Environmental Defense Fund, Friends of the Earth), die aus verschiedensten Motiven heraus sich gegen die Gewährung des Kredites wandten. Als Hauptargument wurde jedoch die geringe Aufmerksamkeit angeführt, die bei der Kreditvergabe den Umweltauswirkungen gegolten hatte. Letztlich wurde die umweltpolitische Frage jedoch der Sicherung der heimischen Kupferindustrie untergeordnet, was daraus ersichtlich ist, daß keinerlei positive Maßnahmen vorgeschlagen wurden, die das mexikanische Projekt in Übereinstimmung mit den US-Luftqualitätsnormen gebracht hätten.49

Der Widerstand seitens der USA konnte die Zuteilung der Kredite nur zeitweise verzögern, gleichzeitig lief jedoch auch eine Kampagne seitens der nordamerikanischen Umweltgruppe Smelter Crisis Project an, die sich gegen die Luftbelastung durch die nordamerikanische Firma in Douglas wandte. Diese wurde für den sauren Regen in der Region verantwortlich gemacht, da sie auf der Basis einer Sondergenehmigung durch die amerikanische Umweltbehörde arbeitete. Im Juli 1986 wurde die Fabrik vorübergehend geschlossen, so daß sich nunmehr das Interesse auf die seit 1983 in Sonora arbeitende Fabrik Nacozari richtete. Vor dem Hintergrund des Agreements, das ja die gemeinsame Wahrnehmung der Umweltinteressen in der $100 \mathrm{~km}$ Zone beiderseits der Grenze vorsieht, gelang es den Umweltgruppen, das Thema der Luftbelastung durch die Kupferproduktion auf die Tagesordnung der gemeinsamen Kommissionen zu setzen. Im Februar 1985 einigen sich beide Parteien auf ein erstes Übereinkommen zur Kontrolle der Emissionen, wobei Mexiko auf eine Erweiterung der Anlage in Cananea ohne den Ausbau entsprechender Filteranlagen verzichtet, gleichzeitig wurde von beiden Seiten die Kontrolle der Produktionsstätten in Nacozari und Douglas zugesagt. Im Januar 1987 unterschrieben beide Regierungen den Anhang 4 zum Agreement, der gemeinsame Normen und Emissionswerte festlegt. Auf die-

48 Vgl. Steven Mumme, The Cananea Copper Controversy: Lessons for Environmental Diplomacy, in: Inter-American Economic Affairs vol. 38/1984, pp. 5 ff.

49 Mumme op. cit., p. 13. 
ser Grundlage sollen Beauftragte beider Parteien im halbjährigen Abstand die Einhaltung der Normen überprüfen, um eine dauerhafte Verbesserung der Luftbelastung im Grauen Dreieck sicherzustellen.

Diese Vereinbarung stellt die erste erfolgreiche Harmonisierung von Umweltstandards dar, deren Vollzug auch von beiden Seiten überwacht wird. Die politischen Intentionen bezogen sich damit nicht so sehr auf die konkrete Kostenzurechnung und die dafür notwendige Bestimmung des Verursachers; vielmehr versuchten beide Parteien das gemeinsame Interesse einer Verhinderung des sauren Regens in der Region anzugehen. Entscheidend für die Lösung der Umweltbelastung war sicherlich auch, daß der mexikanischen Seite hinreichend finanzielle Mittel durch die Weltbank zur Verfügung standen, um die nötigen Investitionen vornehmen zu können. Wie auch im Falle der Abwasserregulierung Tijuanas ging großer Druck von seiten der nordamerikanischen Umweltgruppen aus, die die mexikanische Regierung zur Wahmehmung einer bisher vernachlässigten Umweltproblematik zwangen.

\section{II.1.4. Maquiladora-Industrie und Umweltverträglichkeit}

Am 19. Januar 1967 wird im Diario Oficial, dem Bundesgesetzblattes Mexikos, das Dekret veröffentlicht, das die Einfuhr oder den Export von gefährlichen Materialien und Abfällen ordnet. Diese Bestimmung gilt in besonderem Maße für die sog. Maquiladora-Industrie, jene Lohnveredelungsbetriebe, die sich ganz oder teilweise Exportaktivitäten widmen, zum Gewinn von Devisen beitragen, Arbeitsplätze schaffen und eine ausgewogene regionale Entwicklung fördern sollen.50 Die Umweltbehörde Mexikos schreibt für den Betrieb eine ökologische Erklärung vor, in der die gefährlichen Materialien oder Abfälle benannt und gegebenenfalls genehmigt werden müssen. Darüber hinaus heißt es: "Gefährliche Materialien und Abfälle, die in den Produktionsprozessen, der Transformation oder der Weiterverarbeitung von Rohstoffen, die in das Land nach den Regeln der vorübergehenden Einfuhr gelangt sind, erzeugt werden, müssen in das Herkunftsland zurückgeführt werden." 51 Das schnelle Wachstum der Maquiladora-Industrie mit jährlichen Raten von mehr als $10 \%$, hat die Behandlung gefährlicher Abfälle unkontrolliert werden lassen. Weder seitens der mexikanischen noch seitens der nordamerikansichen Zollbehörden liegen hinreichende Aufzeichnungen darüber vor, wo bisher angefallene Abfälle verblieben sind. Obwohl das Agreement von 1983 im Anhang 2 (1985) die verpflichtende Anmeldung des Transportes gefährlicher Stoffe vorsieht, sind bisher die notwendigen Mitteilungen unterblieben. Es

50 Vgl. das "Dekret zur Förderung und zum Betrieb der exportorientierten Maquiladora-Industrie", veröffentlicht im Diario Oficial vom 15. August 1983, Art. 1.

51 Vgl. René Franco Barreno, Impacto ambiental de la industria maquiladora en la frontera norte de México, in: Arturo Garcia Espinosa (ed.): Maquiladoras. Primera Reunión sobre Asuntos Fronterizos, Nuevo León 1988, S. 96. 
liegt der Verdacht nahe, daß die angefallenen Abfälle entweder in den Betrieben verblieben sind, oder den in Mexiko ansässigen giftmüllverarbeitenden Betrieben angeliefert wurden. Darüber hinaus ist nicht auszuschließen, daß für die Ansiedlung von Maquiladora-Betrieben auch die weniger strikte Umweltgesetzgebung in Mexiko ausschlaggebend sein könnte. Einer Umfrage zufolge betrachten $12 \%$ der Investoren Umweltfaktoren als sehr wichtig für ihre Ansiedlungsentscheidung. 52 Gerade der Widerstand in der amerikanischen Bevölkerung gegen giftmüllverarbeitende Betriebe und Mülldeponien (Right to Know Act sowie der Grundsatz des "not in my backyard") lassen es für nordamerikanische Betriebe interessant erscheinen, ihre Produktionsanlage nach Mexiko zu verlegen.53 Mit dem neuen Reglement für die Kontrolle toxischer Abfälle vom Mai 1989 ist von mexikanischer Seite nunmehr ein wichtiger Schritt erfolgt, um zu einem Inventar der Emissionen dieser Abfälle zu gelangen. Hierfür sind gemeinsame und unangemeldete Besuche der binationalen Beauftragten vor Ort geplant und auch bereits durchgeführt worden, um eine schrittweise Anpassung der Umweltnormen zu erreichen. Gleichwohl ist insbesondere die Behandlung der gefährlichen Abfälle in Mexiko weiterhin ungeklärt, da sich dabei die Interessen der Industrieförderung und des Umweltschutzes überschneiden.

\section{II.2. Die institutionelle Struktur der Umweltaußenpolitik}

Die Beschäftigung der mexikanischen Regierung mit Umweltfragen findet im Jahre 1971 ihren ersten Niederschlag, indem das Bundesgesetz zur Prävention und Kontrolle der Umweltverschmutzung verabschiedet wird. $54 \mathrm{Im}$ Jahr darauf wird das Staatssekretariat für die Verbesserung der Umwelt, das im Gesundheitsministerium angesiedelt ist, eingerichtet. Gerade durch das in den USA sich ausbreitende Umweltbewußtsein und die Notwendigkeit einer altemativen Politik zu den Forderungen der USA im binationalen konsultativen Prozeß, wuchs die Orientierung Mexikos an der nordamerikanischen Environmental Protection Agency (EPA). Im Jahre 1981 fand eine erste Novelle des Umweltgesetzes statt, die als neues Element Vorschriften eines Umweltstrafrechtes enthält. Erst mit dem Regierungsantritt von Miguel de la Madrid im Jahre 1982 erhält die "Okologie" Kabinettsrang, indem die Umweltpolitik mit der Wohnungsbaupolitik in einem gemeinsamen Ministerium, die Secretaría de Desarrollo Urbano y Ecología (SEDUE) zusammengefaßt wird. Das Umweltgesetz Mexikos aus dem Jahre 1988 (La ley general del equilibrio ecológico y la

52 Vgl. Roberto Sánchez, Impacto ambiental de la maquiladora: altemativas para su control, (Vortrag gehalten auf dem Intemationalen Seminar "La maquiladora en México" 5. bis 7. Juni 1989, México, D.F.

$53 \mathrm{Vgl}$. Roberto Sánchez, Contaminación de la industria fronteriza: riesgos para la salud y el medio ambiente, in: Bernardo González-Aréchiga/Rocio Barajas Escamilla (comp.), Las maquiladoras: a juste estructural y desarrollo regional, Tijuana 1989, S. $158 \mathrm{ff}$.

$54 \mathrm{Vgl}$. Reglamento para la prevención y el control de la contaminación atmosférica originada por humos y polvos. 
protección al ambiente) faßt die zentralen Vorschriften zur Bewahrung und Restaurierung des ökologischen Gleichgewichtes zusammen und bildet somit die Grundlage für die staatlichen Ausfuhrungsbestimmungen und Normsetzungen, die bislang nur langsam voranschreiten.

Der politische Ansatz der mexikanischen Umweltpolitik liegt dabei weniger in der Kontrolle durch Verbote etc. begründet, vielmehr wird versucht, durch planerische Gestaltung und Aushandlungsprozesse die notwendige Anpassung zwischen SEDUE und parastaatlichen Industrien bzw. der Privatwirtschaft einzuleiten. Aufgrund des noch weithin an die Mittelschichten gebundenen Umweltbewußtseins sind bisher wenig Impulse von der Umweltbewegung ausgegangen, die sich in Teilbereichen organisiert hatte.55 Wegen der geringen institutionellen Ausstattung bleibt die mexikanische Umweltpolitik auch weiterhin einem Vollzugsdefizit verhaftet, bzw. muß sich auf symbolische Politik beschränken.56

Demgegenüber hat sich in den USA eine vielgestaltige Umweltbewegung entwickelt, die durch Hearings und öffentlichkeitswirksame Maßnahmen die Regierung unter Druck zu setzen weiß. Daneben verfügen die USA mit der EPA über eine institutionell und professionell gut aufgebaute Organisation, welche eine umfassende Kontrolle von Auflagen sicherstellen kann. Allerdings unterliegt auch die Umweltpolitik in den USA der politischen Einflußnahme in konkreten Fällen, in denen die ökonomische Entwicklung der ökologischen Sicherheit übergeordnet wird.

Betrachtet man diese institutionellen Elemente und interpretiert ihre Auswirkungen auf die inhaltliche Füllung einer Umweltaußenpolitik, so wird sehr schnell deutlich, daß Mexiko eine eher passive Rolle im bilateralen Aushandlungsprozeß eingenommen hat. Der Druck, der seitens der USA ausgeübt wurde, führte zu einer nationalistischen Reaktion in Mexiko, womit die gemeinsame Lösung der angesprochenen Probleme behindert wurde. Der Versuch der USA, grundsätzlich das Verursacherprinzip durchsetzen zu wollen, wie dies in besonderem $\mathrm{Maße}$ im Fall der Abwasserregulierung Tijuanas gezeigt wurde, hat sich als Hindernis für eine technisch sinnvolle Lösung langfristiger Art erwiesen. Mexiko ließ sich unter dem Druck, seine Außenbeziehungen zu den USA nicht durch Umweltprobleme beeinträchtigen zu lassen, auf Entscheidungen ein, die weder sachgerecht noch politisch zukunftsweisend sind.

Die konkrete Verhandlungsfuhrung war seitens der USA darauf ausgelegt, eine Fall-zuFall-Strategie zu verfolgen, während Mexiko an einem politischen Gesamtkonzept interessiert war. Das Agreement von 1983 stellt in dieser Hinsicht einen Erfolg Mexikos dar, der

55 Vgl. Stephen Mumme/Richard Bath/Valerie Bassetto, Political Development and Environmental Policy in Mexico, in: Latin American Research Review vol. 23/1988, p. 20.

56 Ibid., p. 23. 
jedoch durch die jeweils neu angefügten Anhänge wieder aufgeweicht wurde. Der Versuch Mexikos zu einem package dealing zu gelangen, um damit die asymmetrische Struktur seiner Außenbeziehungen zu den USA ausgleichen zu können, war bisher nicht grundsätzlich durchzusetzen. Es gelang Mexiko nur, in den Fällen gefährlicher Abfälle und der Lösung der Luftbelastung durch die Kupferproduktion im Grauen Dreieck ein Konzept der gemeinsamen Wahmehmung von Umweltinteressen einzubringen, das sich zum einen auf die Festlegung verbindlicher Standards und zum anderen auf die jüngst erstmals praktizierten Besuche in Maquiladora-Betrieben durch Beauftrage von EPA und SEDUE bezieht. Dieser Teilerfolg läßt sich wohl vor allem auch darin erkennen, daß es sich in beiden Bereichen um Sektoren handelt, die auch für die nordamerikanischen Wirtschaftsinteressen von Bedeutung sind. Die Definition einer Umweltaußenpolitik erwies sich eben dort als sinnvoll, wo der Interdependenz auf wirtschaftlichem Gebiet der politische Wille (sei es durch Druck ökonomischer oder politischer Interessen) entsprach. Darüber hinaus versäumte es Mexiko, eine Stärkung seiner Position durch direkte Kontakte zu gesellschaftlichen Gruppen der USA zu suchen, so daß die Vorschläge von mexikanischer Seite ausschließlich von der Regierung vorgetragen wurden bzw. sie der einzige Ansprechpartner der USA war.

Allerdings etablierte sich eine Staatsgrenzen überschreitende interkommunale Zusammenarbeit, die in ein Programm der "Zwillingsstädte" Tijuana und San Diego sowie Ciudad Juárez und El Paso mündete. Die gemeinsamen Anstrengungen zu einer übergreifenden Raumordnung und Landesplanung haben sich trotz der zentralistischen Struktur Mexikos durchgesetzt und eine erfolgreiche Mikrodiplomatie begründet, die sich mit der Ansiedlung der Maquiladora-Industrie noch verstärkt hat. Gemeinsame Handelskammem, Unternehmervereinigungen etc. haben diesen Proze $ß$ weiter vorangetrieben und damit eine sachbezogene technische Zusammenarbeit gefördert, die den Charakter "weicher" Umweltpolitik noch deutlicher erkennen läßt.

\section{Außenpolitik und Völkerrecht - Wege zur grenzüberschreitenden Umweltpoli- tik?}

Der Mißerfolg der internationalen Umweltbeziehungen hat zu der These geführt, die Rolle des Nationalstaates müsse auch im Bereich der Umweltpolitik stärker in Rechnung gestellt werden. Es ist sicherlich unbestreitbar, daß der Nationalstaat nach wie vor als entscheidende Größe in der internationalen Politik anzusehen ist. Gerade die Tatsache, daß er seine als undurchdringbar angesehene Schale 57 nicht mehr bewahren kann, wird am Gegenstand der grenzüberschreitenden Umweltprobleme deutlich. Das unmittelbare Interesse nationalstaatlicher Politik, mögliche Umweltschäden vom eigenen Territorium fernzuhalten, verweist, zumindest kurzfristig betrachtet, auf die Verlagerung entsprechender Umweltfolgen 
auf die Nachbarstaaten, soweit nicht ein Export von umweltgefährdenden Stoffen und Produktionsverfahren in die Dritte Welt als möglich erscheint. Andererseits kann der Nationalstaat die Auswirkungen schlechter Umweltqualität in den Nachbarstaaten auf das eigene Territorium nicht kontrollieren. Der klassische Fall der Externalisierung von Umweltlasten und Umweltschutzkosten beinhaltet den Interessengegensatz von Nationalstaaten, die durch grenzüberschreitende Umweltprobleme in einer Zwangsgemeinschaft verbunden sind. Die Beschwörung gemeinsamer Interessen in der Umweltpolitik über nationalstaatliche Grenzen hinaus ist jedoch solange nur als Programmsatz zu verstehen, wie keine konkrete Verteilung von Nutzungsrechten und Umweltschutzkosten vorgenommen wird. Gerade an diesem Punkt geraten sowohl Umweltvölkerrecht wie auch Umweltaußenpolitik an ihre Grenzen, verfügen beide doch weder über eine Instanz zur Kontrolle des Vollzuges noch über Mechanismen der Garantie ökologischer Sicherheit im Rahmen der nationalen Infrastruktur. Die Hilfshypothese, Umweltaußenpolitik sei nur dann glaubwürdig und erfolgreich, wenn sie innenpolitisch durch ein ausgebildetes Umweltbewußtsein abgedeckt ist, verliert dann an Gültigkeit, wenn es den beteiligten Nationen aufgrund von Ressourcenmangel unmöglich ist, international vorgegebene Standards zu erfüllen. In diesem Sinne stellt das Streben nach der Harmonisierung der Umweltnormen zwar eine wichtige Voraussetzung dar, gleichzeitig bleibt jedoch die Wirkung solcher Vereinbarungen zweifelhaft, wenn das Verursacherprinzip zum grundsätzlichem Maßstab grenzüberschreitender oder globaler Umweltprobleme erhoben wird. In vielen Fällen wird eher das Gemeinlastprinzip im Sinne einer Gemeinwohlzielsetzung eine befriedigende Lösung von Umweltproblemen erlauben und daher zum Charakteristikum intemationaler Umweltpolitik werden müssen. Die indirekte Internationalität des Umweltschutzes, die sich aus den Wirkungen des internationalen Wirtschaftsrechtes auf das Umweltschutzrecht ergibt,58 verweist ebenso auf diesen Zusammenhang.

Damit wird emeut die idealistische Komponente in den Vordergrund gerückt, die schon im Funktionalismus angesprochen wurde mit dem Slogan: From rights to services. 59 Die erfolgreiche Durchsetzung einer internationalen Umweltpolitik wird sich daher nur aus der Berücksichtigung der Aktorenvielfalt und der Interdependenz in der internationalen Politik ergeben können, die zwar dem Nationalstaat seinen Platz einräumt, aber gleichzeitig auch der aus der bilateralen Abkommen und der Struktur der internationalen Politik entstehenden Ordnung die notwendige Aufmerksamkeit zukommen läßt.

58 Vgl. Oppermann, Gesetzte Normen ..., op. cit., S. 7.

59 Vgl. Mitrany, op. cit., S. 17. 


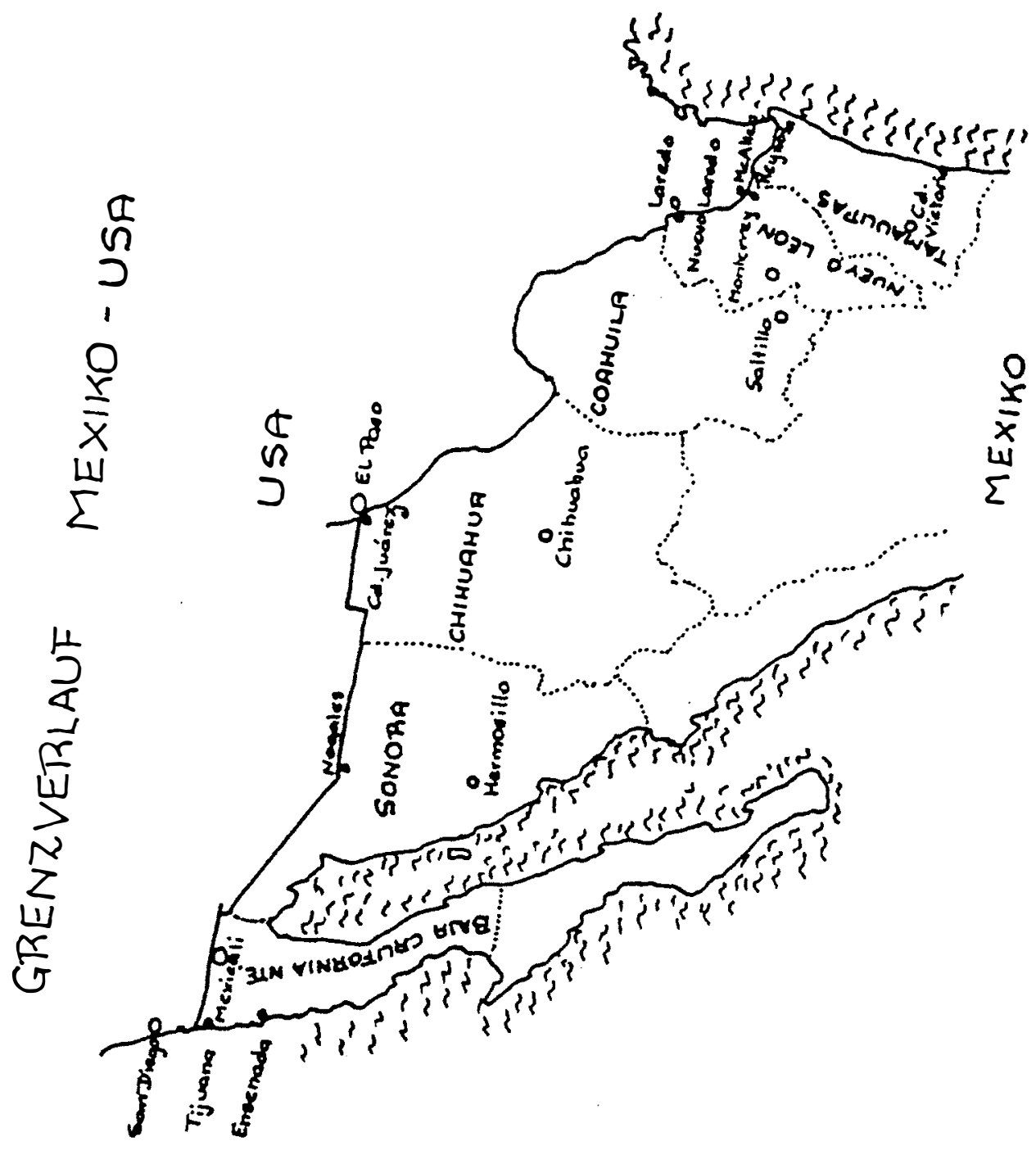




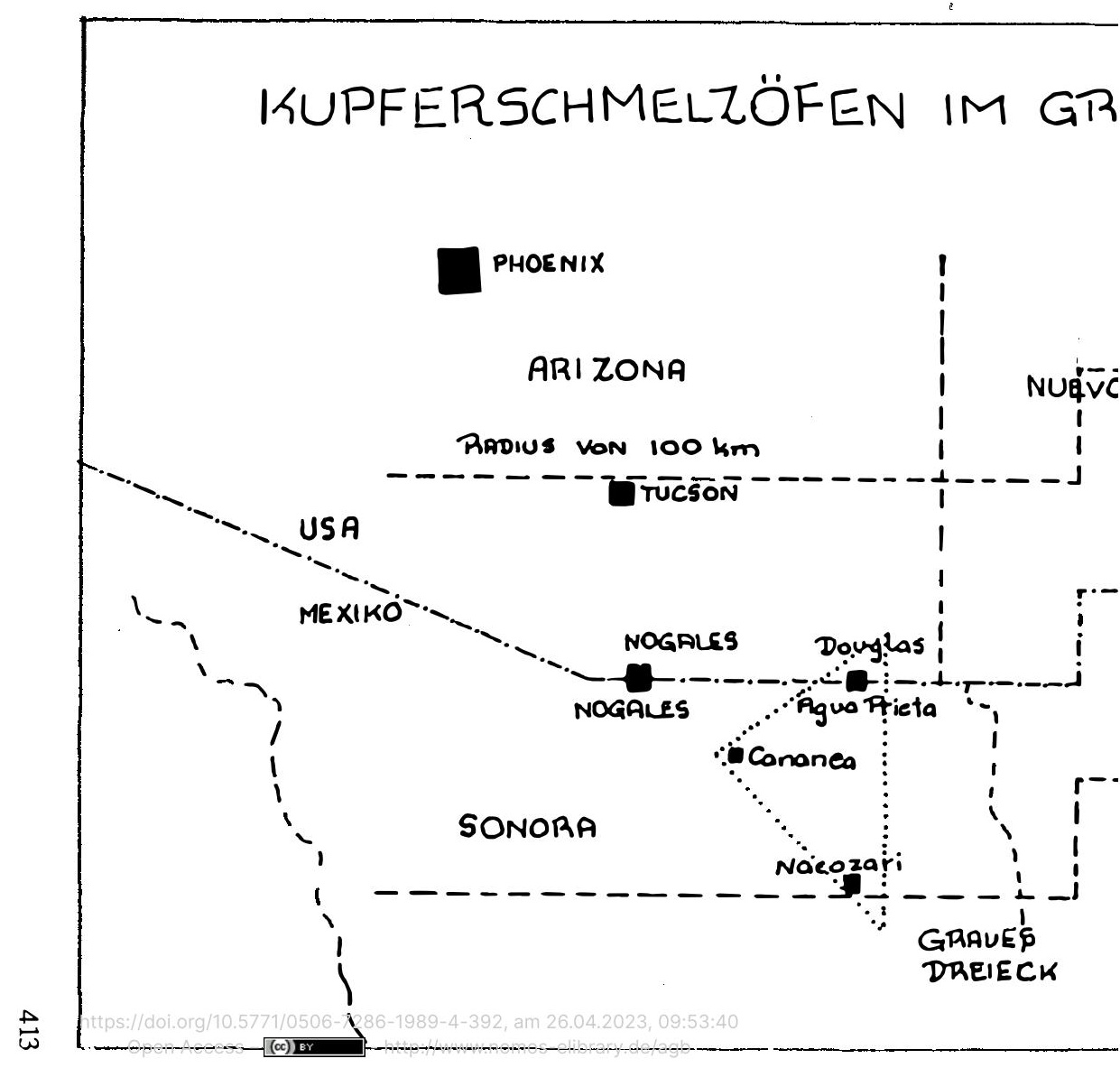




\title{
ABSTRACTS
}

\section{Fighting Transboundary Waste Streams: Will the Basel Convention help?}

\author{
By Susanne Rublack
}

The increasing occurrence of hazardous waste exports from industrialized countries mainly to African countries has coincided with efforts on the international level to create norms which subject such transactions to controls. The first binding instrument on transboundary movements of hazardous wastes was adopted at the Basel diplomatic conference in March of 1989. Since the African countries refused the signature of the Basel Convention, because in their view it does not go far enough to prevent incidents of waste dumping in the developing world, it is still uncertain what contribution to a co-operative solution of the problem this instrument will be able to make. The article attempts a first analysis of the convention's main elements and gives some background on its evolution, on the phenomenon of international waste trade itself and on other related regulatory initiatives.

Although the provisions of the treaty lack stringency on some central issues, they may in their overall substance be viewed as a constructive step towards controlling environmental dangers which the disposal or treatment of hazardous waste generates in particular in developing countries.

\section{Environmental Problems as Topics of Foreign Policy: Mexico - USA}

\section{By Günther Maihold}

Due to the impact of the United Nations Conference on the Human Environment at Stockholm in 1972, a tendency evolved in international environmental policy to favour international organizations as main actors in the political efforts to advance in the regulation of environmental concems on a world-wide scale. As these organizations were unable to perform an effective role in this area, a shift in environmental policy has been proposed that could be able to provide new impetus for international environmental law and a solution for the consequences of international interdependence. 
This proposal is reviewed in the context of Mexican-US-relations with the intention to discuss the concrete problems of bi-national negotiations in the case of the Colorado River salinity problem, the waste water of Tijuana and San Diego, the Cananea Copper controversy and the hazardous waste in the maquiladora-industry. These specific issues in the borderlands present different styles of negotiation; they are examined in order to gain results for the general question whether principles like "the polluter pays" can be applied in the relations between industrial and developing nations which are immersed in an asymmetrical interdependence.

The article emphasizes the impact of "micro-diplomacy" in the borderlands which gives the people concerned and involved with the local problems the chance to develop an environmental policy across the border. Bilateral package agreements will allow the Mexican government a more effective environmental negotiation. Meanwhile, the United States will continue with their case-by-case approach. The definition of the costs of environmental policies on a global scale will only be possible, if a common-good perspective is widely accepted which offers all nations a real chance to participate in the solution of future global problems.

\section{The Protection of the Tropical Rain Forests by Way of Economic Compensation}

\section{By Dieter Oberndörjer}

The article gives a closer insight into the background of the destruction of the tropical rain forests. The author not only reports on the present status quo of the forests, but also tries to give an overview over the effects and causes of this large scale destruction of tropical rain forests. It becomes clear that a wide spectrum of elements is responsible for the complex situation of the rain forests. Economic, socio-cultural, biological and environmental aspects are being discussed.

The author then proceeds to review and criticize the concept of sustainable development and its implications on the critical situation of the rain forests. Various contradictory aspects of this concept are revealed and discussed. The Tropical Forest Action Plan of the FAO which is subsequently surveyed does, in the eyes of the author, not qualify as a sound solution for the issue of deforestation, either.

Finally, the author describes the concept of protection of tropical forest areas by way of economic compensation. He illustrates the opportunities that lie within the concept's soft 